\title{
Automatic Identification of Frankfurt Plane and Mid-Sagittal Plane of Skull
}

\author{
Yuan Cheng, Wee Kheng Leow \\ Dept. of Computer Science \\ National University of Singapore \\ \{cyuan, leowwk\}@ comp. nus.edu.sg
}

\author{
Thiam Chye Lim \\ Dept. of Surgery \\ National University Hospital \\ thiam_chye_limenuhs.edu.sg
}

\begin{abstract}
Automatic identification of the Frankfurt plane (FP) and mid-sagittal plane (MSP) of a human skull is a very important task. These planes are used to define important landmarks on the skull for surgery planning and guidance, forensic reconstruction, and anthropological and archaeological studies. At present, there is no known method for automatic identification of FP. Existing methods identify FP by fitting a plane over manually marked landmark points. On the other hand, existing automatic methods identify MSP by looking for a symmetric plane that divides the skull into the left and right sides. Unfortunately, this approach is inaccurate because extensive anthropological studies show that there is significant lateral asymmetry in the skulls. This paper proposes an automatic, accurate, and robust method for identifying FP and MSP of skulls. It registers a template skull model with known landmarks to a target skull to locate the landmarks, FP, and MSP on the target skull. Then, it iteratively refines the landmark locations, FP and MSP according to their medical definitions. Test results show that the proposed algorithm is more robust and accurate than symmetry-based methods. Moreover, it can also be applied to partial skull models of real patients.
\end{abstract}

\section{Introduction}

In surgery, forensics, and anthropology, identification of the Frankfurt plane (FP) and mid-sagittal plane (MSP) of a human skull (Fig. 1) is a very important task. These anatomical planes are used to define the three anatomical orientations of the skull: lateral (left-right), anteriorposterior (front-back), and superior-inferior (up-down). These anatomical orientations, in turn, are used to define craniometric landmarks that are used for preoperative surgery planning, intraoperative surgery guidance, forensic reconstruction, and anthropological and archaeological studies $[4,6,8,12,13,15]$. Thus, automatic identification of FP and MSP, and landmarks, greatly facilitates computerassisted processing and analysis in these applications.
The skull is a very complex 3D structure consisting of 28 bones that are fused together. While its general shape is similar for all normal humans, it can vary greatly in size and shape details among different individuals. Old age, diseases, and injuries can cause further changes to the skull's shape. Therefore, automatic identification of FP and MSP is a very difficult and challenging task.

At present, there is no known method for automatic identification of FP. Existing methods require the user to manually mark landmark points of FP on the skull and then fit a plane over the landmark points [8, 9]. This straightforward approach requires an experienced user to accurately locate the landmark points. On the other hand, existing automatic methods for identifying MSP look for a symmetric plane of the skull [6,14]. Unfortunately, this approach is inaccurate because extensive anthropological studies show that there is significant lateral asymmetry in the human skulls [11, 15]. In anatomy, MSP is in fact defined not as a laterally symmetric plane of the skull but as a vertical plane that passing through the midline of the skull [1], and the midline is defined by specific landmarks on the skull.

This paper proposes an automatic, accurate, and robust method for identifying FP and MSP of 3D skull models. The key idea is to accurately locate anatomical landmarks that define FP and MSP. But, these landmarks are defined according to the skull's anatomical orientations, which are, in turn, defined by FP and MSP. To resolve this difficulty, our proposed method registers a template skull model with known landmarks to a target skull to automatically locate the landmarks on the target skull. It then fits two planes to the landmarks to obtain good initial estimates of FP and MSP. Then, it iteratively refines the locations of the landmarks and the locations and orientations of FP and MSP. Test results show that the proposed algorithm is more robust and accurate than symmetry-based methods. Moreover, it can also be applied to partial skull models of real patients.

\section{Related Work}

As discussed in the previous section, there is currently no automatic method for identifying FP. On the other hand, 


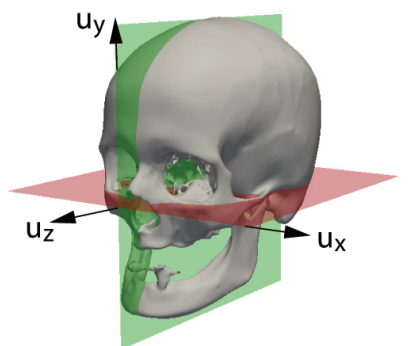

(a)

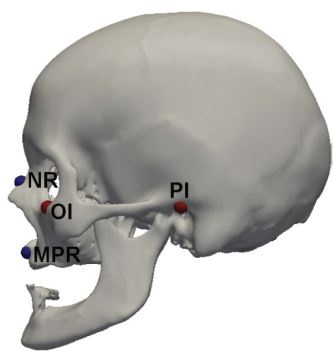

(b)

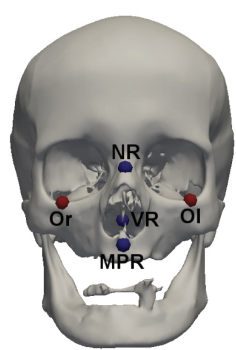

(c)

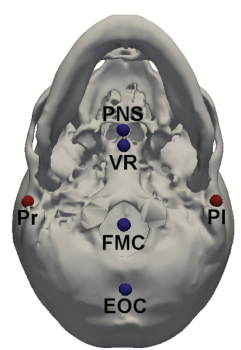

(d)

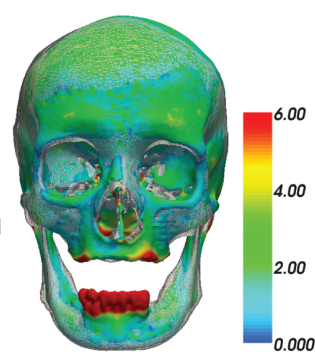

(e)

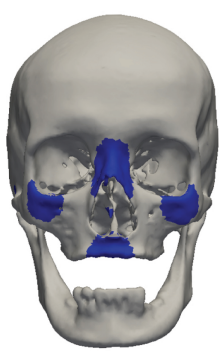

(f)

Figure 1. Skull models. (a) Frankfurt plane (FP) is the horizontal (red) plane and mid-sagittal plane (MSP) is the vertical (green) plane. (b-d) Template model. Red landmarks define FP and blue landmarks define MSP. (e) FICP registration result of target model. Colors indicate the distances of the target points to corresponding template points. (f) Landmark regions (blue) are located on the target model.

there are existing methods for automatic or semi-automatic identification of MSP [6, 14]. In the work of [6], two methods were proposed. The first method requires the user to indicate the points on the MSP and/or laterally symmetric points on both sides of the skull. Then, it fits a plane over the points as the MSP. The second method requires the user to indicate laterally symmetric parts on both sides of the skull. Then, it determines the mirror reflection plane between the left and right parts, which is regarded as the MSP.

The method of [14] requires the user to indicate the regions that contain the landmark points that define the MSP and a transverse plane that is orthogonal to MSP. Then, it detects extreme points in these regions as landmark points, and fits two orthogonal planes through these points. The fitted vertical plane is regarded as the MSP.

Identifying MSP based on lateral symmetry is inaccurate because extensive anthropological studies show that there is significant lateral asymmetry in the human skulls $[11,15]$. Another shortcoming of existing methods is the manual marking of landmark points or regions on the skull. This approach is tedious and it requires an experienced user to locate the landmarks accurately.

In comparison, our proposed method is not based on lateral symmetry. Instead, it detects midline features that are used in anatomy to define MSP. Moreover, it automatically detects landmarks for identifying FP and MSP.

\section{Anatomical Landmarks}

In anatomy, the Frankfurt plane (FP) is defined as a plane that passes through the orbitales and the porions [1] (Fig. 1). The left and right orbitales (Ol, Or) are the lowest points of the lower margin of the left and right orbits (eye sockets). The left and right porions ( $\mathrm{Pl}, \mathrm{Pr})$ are the most lateral points of the roofs of the left and right ear canals.

The mid-sagittal plane is defined as a vertical plane that passes the midline of the skull [1]. A number of features lie on the skull's midline. Based on the landmarks used in anatomy [12] and forensics [13], 6 landmarks are selected to define the midline (Fig. 1):
- The nasal bone suture (NR) is a ridge structure formed by the joint of the left and right nasal bones.

- The mid-philtrum ridge (MPR) is a ridge structure along the anterior (front) nasal spine towards the upper lip margin.

- The posterior nasal spine (PNS) is the peak at the posterior end of the median palatine suture, which is the joint of the left and right palatine bones.

- The vomer ridge (VR) is a ridge structure at the vomer, which forms a part of the nasal septum (the bone that divides the nose into left and right airways).

- The foramen magnum center (FMC) is the center of foramen magnum, a circular opening at the bottom of the skull where the spine cord passes through.

- The external occipital crest (EOC) is a ridge structure along the midline at the bottom of the skull.

The ridges lie on or close to MSP. So, any point on a ridge can be used as a landmark that represents the ridge for defining MSP. In the current implementation, an estimate of the ridge's centroid is used as the ridge landmark point.

\section{FP and MSP Identification Algorithm}

In anatomy, FP and MSP are used to define the skull's anatomical orientations (Fig. 1a). In particular, lateral orientation is normal to MSP and up-down orientation is normal to FP. These orientations, in turn, are used to define craniometric landmarks. For example, the orbitale is the lowest point of the orbit. But the lowest point changes as the orbit is rotated. So, incorrect skull orientation can result in inaccurate localization of landmark points, which in turn leads to inaccurate identification of MSP and FP that define the skull orientation. To resolve this difficulty, an iterative algorithm is required to iteratively refine the estimations of the landmarks, MSP and FP.

An overview of the iterative algorithm is given below:

\section{FP and MSP Identification Algorithm}

1. Register a template mesh model with known landmarks to the target mesh model (Section 4.1). 
2. Locate the landmarks on the target model using the registered template model, and fit FP and MSP to the landmarks (Section 4.2). These landmarks and the fitted planes serve as the initial estimates.

3. Repeat until convergence:

(a) Refine the locations of the FP landmarks, and fit FP to the refined FP landmarks (Section 4.3).

(b) Refine the locations of the MSP landmarks, and fit MSP to the refined MSP landmarks, keeping it orthogonal to FP (Section 4.4).

\subsection{Model Registration}

Human skulls naturally vary in shape details and sizes. Moreover, parts of the target skull may be missing due to diseases or injuries. To achieve good registration under these conditions, Fractional Iterative Closest Point (FICP) [10], a variant of ICP [3] robust to these variations, is used. Like ICP, FICP iteratively computes the best similarity transformation that registers the template to the target. The difference is that in each iteration, FICP computes the transformation using only a subset of template points whose distances to the target model are the smallest. Fig. 1e shows that FICP registration is very robust and accurate despite the differences between the template and the target.

\subsection{Initialization}

After registering the template model to the target model, known landmark points on the template are located on the target model. This is achieved by mapping the template landmarks to the corresponding points on the target. Given these initial estimates of the landmark points, two planes are fitted to these points to yield the initial estimates of FP and MSP by applying Principal Component Analysis (PCA). The mean of the landmark points gives the position of the plane, and the smallest eigenvector obtained by PCA gives the unit normal vector of the plane. So, the FP and MSP are each represented by its position and unit normal vector.

The initial FP and MSP define initial estimates of the skull's orientations. Let $\mathbf{u}_{x}, \mathbf{u}_{y}$, and $\mathbf{u}_{z}$ denote the unit vectors pointing towards the left-lateral, up, and front directions (Fig. 1a). Then, $\mathbf{u}_{x}$ is normal to MSP, $\mathbf{u}_{y}$ is normal to FP, and $\mathbf{u}_{z}=\mathbf{u}_{x} \times \mathbf{u}_{y}$.

Due to normal variations of the human skulls, the template and target models may differ in shape details and sizes. So, the initial estimates of the target landmark points are not accurate. To obtain more accurate estimates, an elliptical region is placed around each target landmark point, and the mesh surfaces within the elliptical region are identified as the landmark region. The orientation and size of the ellipse are empirically predefined to fixed values, and they vary for different landmarks according to the shape of the skull around the landmark. It should be large enough to

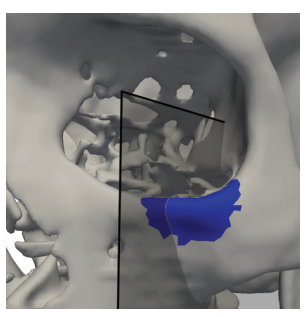

(a)

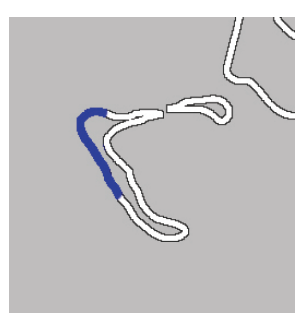

(b)

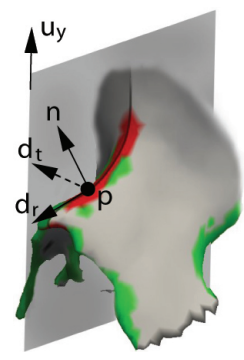

(c)
Figure 2. Sagittal section and ridge structure. (a) Sagittal section of lower orbit is indicated by the gray plane. (b) A sagittal section. Landmark region is colored blue. (c) Colored regions around the ridge have large curvatures, but only the red region is on the ridge.

include the landmark on the target model. During iterative refinement (Section 4.3, 4.4), accurate locations of the landmarks are searched within the landmark regions according to the definitions of the landmarks. An exception is FMC, the center of foramen magnum. Since FMC does not lie on skull surface, landmark region is not defined for FMC.

\subsection{Frankfurt Plane Identification}

The Frankfurt plane (FP) passes through the orbitales and the porions (Fig. 1). The orbitales (Ol, Or) are the lowest points of the lower margins of the orbits. To accurately locate the (left or right) orbitale, sagittal sections $S(x)$ of the lower margins parallel to the MSP are obtained, where $x$ is a local coordinate along the $\mathbf{u}_{x}$ direction. Each sagittal section $S(x)$ contains a part of the landmark region $R$ of the orbitale (Fig. 2). The points $\mathbf{q}(x)$ on the lower margins correspond to the highest points in the intersections between $R$ and $S(x)$. The orbitale is the lowest point $\mathbf{q}(x)$ in the $\mathbf{u}_{y}$ direction. In other words, its $x$-coordinate minimizes the following objective function $F(x)$ :

$$
F(x)=\mathbf{q}(x) \cdot \mathbf{u}_{y}, \quad \mathbf{q}(x)=\arg \max _{\mathbf{p} \in R \cap S(x)} \mathbf{p} \cdot \mathbf{u}_{y} .
$$

Porions are the most lateral points on the roof of the external bony ear holes. The surface normals of the roof points are in the direction of $-\mathbf{u}_{y}$. To accurately locate the porions, the algorithm looks for the most lateral points in the left and right landmark regions $R_{l}$ and $R_{r}$ whose surface normals are close to $-\mathbf{u}_{y}$. That is, select subsets $S_{l}$ and $S_{r}$ of points respectively in $R_{l}$ and $R_{r}$ whose surface normals are close to $-\mathbf{u}_{y}$. Then, the left porion is located at the most lateral point $\mathbf{q}_{l}$ on the left side:

$$
\mathbf{q}_{l}=\arg \max _{\mathbf{p} \in S_{l}} \mathbf{p} \cdot \mathbf{u}_{x},
$$

and the right porion is located at the most lateral point $\mathbf{q}_{r}$ on the right side:

$$
\mathbf{q}_{r}=\arg \max _{\mathbf{p} \in S_{r}} \mathbf{p} \cdot\left(-\mathbf{u}_{x}\right) .
$$

After refining FP landmark points' locations, a plane is fitted to the points using PCA to yield the refined FP. 


\subsection{Mid-Sagittal Plane Identification}

The mid-sagittal plane (MSP) passes through 6 landmarks discussed in Section 3. Four of them are ridges and one is a peak point. So, methods for detecting ridges and peaks on the skull model are required. In theory, these features can be detected by computing curvatures on the skull surface. However, the accuracy of curvature computation is sensitive to the regularity and resolution of the mesh model [5]. So, we apply the method proposed by Avants et al. [2] to compute curvature based on the Gauss map in a local neighborhood, which is numerically more stable.

\subsubsection{Computing Principal Curvatures}

The Gauss map $\mathbf{N}(u, v)$ is a function of the surface normal parameterized by local coordinates $(u, v)$ on the surface. The derivative $d \mathbf{N}$ measures local changes of $\mathbf{N}(u, v)$, which is related to surface curvature. The derivatives $\mathbf{N}_{u}$ and $\mathbf{N}_{v}$ in the $u$ - and $v$-direction lie in the planes formed by the tangents $\mathbf{T}_{u}$ and $\mathbf{T}_{v}$, and they can be expressed as

$$
\mathbf{N}_{u}=a \mathbf{T}_{u}+c \mathbf{T}_{v}, \quad \mathbf{N}_{v}=b \mathbf{T}_{u}+d \mathbf{T}_{v},
$$

for some values $a, b, c$, and $d$. The Jacobian of $d \mathbf{N}$ expressed in the local coordinates gives the shape operator $S$ :

$$
S=\left[\begin{array}{ll}
a & c \\
b & d
\end{array}\right] .
$$

It has been shown that the eigenvalues $\kappa_{1}, \kappa_{2}$ and eigenvectors $\mathbf{e}_{1}, \mathbf{e}_{2}$ of $S$ give the magnitudes and directions of the principal curvatures [7].

In numerical computation, the Gauss map can be approximated by a degree-one polynomial:

$$
\mathbf{N}(u, v)=\mathbf{g}_{0}+\mathbf{g}_{1} u+\mathbf{g}_{2} v .
$$

Fitting Eq. 6 to a neighborhood of points and their surface normals gives the vectors $\mathbf{g}_{i}$. The derivatives $\mathbf{N}_{u}$ and $\mathbf{N}_{v}$ are simply $\mathbf{g}_{1}$ and $\mathbf{g}_{2}$. Then, the principal curvatures $\kappa_{1}, \kappa_{2}$ and their directions $\mathbf{e}_{1}, \mathbf{e}_{2}$ can be computed from $\mathbf{N}_{u}$ and $\mathbf{N}_{v}$ using the method discussed above.

\subsubsection{Ridge Detection}

Landmark points NR, MPR, VR, and EOC are ridges on the skull (Section 3, Fig. 1). A point $\mathbf{p}$ on a ridge that runs along direction $\mathbf{d}_{r}$ has locally maximum curvature along the direction $\mathbf{d}_{t}$ orthogonal to $\mathbf{d}_{r}$. Since the ridge lies in MSP, the ridge direction $\mathbf{d}_{r}$ and surface normal $\mathbf{n}$ at $\mathbf{p}$ lie in the same plane as $\mathbf{u}_{y}$ (Fig. 2c), which has been refined in Step 3(a) of the algorithm (Section 4.3). So, $\mathbf{d}_{t}$, which is orthogonal to $\mathbf{d}_{r}$, can be computed as

$$
\mathbf{d}_{t}=\frac{\mathbf{n} \times \mathbf{u}_{y}}{\left|\mathbf{n} \times \mathbf{u}_{y}\right|} .
$$

Given a landmark region $R$, the ridge is detected as follows. First, the principal curvatures $\kappa_{1}, \kappa_{2}$ and their directions $\mathbf{e}_{1}, \mathbf{e}_{2}$ are computed using the method discussed in Section 4.4.1. Applying Euler's theorem, their normal curvatures $K_{n}$ along $\mathbf{d}_{t}$ can be computed as

$$
K_{n}=\kappa_{1} \cos ^{2} \phi+\kappa_{2} \sin ^{2} \phi
$$

where $\phi$ is the angle in the tangent plane measured counterclockwise from the direction $\mathbf{e}_{1}$ of the minimum principal curvature to $\mathbf{d}_{t}$. The points with large normal curvature $K_{n}$ are more likely to lie on the ridge.

In general, the triangular faces of a mesh model can vary in size. So, for accurate ridge detection, the high-curvature points are sampled from the centers of triangles instead of mesh vertices. Then, the areas of the triangles can be used as weights of the high-curvature points in ridge detection.

Note that the landmark region $R$ needs to be large enough to include the ridge. As a result, it may also include other nearby surfaces. So, the high-curvature points obtained above may include points on the ridge (inliers) and points on other nearby surfaces (outliers) (Fig. 2c).

RANSAC is applied to iteratively identify the optimal set of inliers. A line $l$ is fitted to the inliers to represent the ridge by applying PCA. The position of $l$ is given by the mean of the inliers weighted by the areas of their triangular faces, and the direction of $l$ is given by the component $\mathbf{u}_{l}$ of the largest eigenvector $\mathbf{v}$ that is parallel to MSP:

$$
\mathbf{u}_{l}=\mathbf{v}_{l} /\left\|\mathbf{v}_{l}\right\|, \quad \mathbf{v}_{l}=\mathbf{v}-\left(\mathbf{v} \cdot \mathbf{u}_{x}\right) \mathbf{u}_{x},
$$

where $\mathbf{u}_{x}$ is the normal vector of MSP. As the triangles typically vary in size, the area-weighted mean yields a more accurate position of the line than the unweighted mean.

\subsubsection{Peak Detection}

Posterior nasal spine (PNS) is a peak landmark point (Section 3). A peak point has a locally maximum Gaussian curvature. To locate the peak accurately, the algorithm computes Gaussian curvature not only for mesh vertices but also for points inside the triangular faces.

A point in a triangle can be uniquely represented by the barycentric coordinates $(u, v)$ defined on the triangle. Then, the method discussed in Section 4.4.1 can be used to compute the principal curvatures $\kappa_{1}(u, v)$ and $\kappa_{2}(u, v)$. The Gaussian curvature is simply $K(u, v)=\kappa_{1}(u, v) \kappa_{2}(u, v)$. So, accurate location of the peak can be computed by finding the position $(u, v)$ over all the triangles in the landmark region $R$ that maximizes the Gaussian curvature $K(u, v)$.

\subsubsection{Foramen Magnum Center Detection}

The margin of the foramen magnum is approximately circular. It has locally maximum curvature along the radial direction. So, to locate the foramen magnum center (FMC), a 
circle $C(\mathbf{c}, r)$ located at $\mathbf{c}$ with radius $r$ is fitted to the FMC region that has large maximum principal curvature $\kappa_{2}$. This is achieved by determining the circle $C(\mathbf{c}, r)$ that minimizes the objective function $F(\mathbf{c}, r)$ :

$$
F(\mathbf{c}, r)=\sum_{\mathbf{p} \in C(\mathbf{c}, r)}\left\{\kappa_{M}-\kappa_{2}[f(\mathbf{p})]+\gamma\|\mathbf{p}-f(\mathbf{p})\|\right\}
$$

where $f(\mathbf{p})$ is the closest point of $\mathbf{p}$ on the target model, $\gamma$ is a constant weight and $\kappa_{M}$ is a constant representing the maximum possible $\kappa_{2}$ value. The first term is minimized for points with large $\kappa_{2}$, and the second term is minimized for points close to $C(\mathbf{c}, r)$.

\subsubsection{Mid-Sagittal Plane Refinement}

Refinement of MSP is performed by fitting a plane to the MSP landmarks subject to the constraint that the plane is orthogonal to FP. Since MSP is orthogonal to FP, its projects to a line $l$ on FP. The perpendicular distance of a point $\mathbf{p}$ to MSP is equal to the distance of the projection of $\mathbf{p}$ on FP to the line $l$. So, the line $l$ can be obtained by fitting it to the projections of MSP landmark points on FP. Then, the midpoint of $l$ gives the location of MSP, and MSP's normal vector $\mathbf{u}_{x}$ can be obtained as the cross product of FP's normal vector $\mathbf{u}_{y}$ and $l$ 's unit direction vector $\mathbf{u}_{z}: \mathbf{u}_{x}=\mathbf{u}_{y} \times \mathbf{u}_{z}$.

\section{Experiments and Discussion}

Four full skulls were used in the experiments, of which three were from Visible Human Project, and one was from OsiriX. One full skull was used as the template (Fig. 1b) and the others were the test targets. In clinical practice, CT images are acquired only for the parts of the skulls under treatment. For this reason, the 3 target full skulls were cut at the top and the bottom to produce 3 additional partial skulls for testing. The only requirement was that all the FP and MSP landmarks could still be located on the partial skulls. Moreover, three partial skulls of patients from a local hospital were also used for testing.

The resolutions of the CT images of the skull models ranged from 0.47 to $1 \mathrm{~mm} /$ pixel. The $\mathrm{CT}$ images were segmented and 3D mesh models were reconstructed from them.

The proposed automatic FP and MSP identification algorithm was applied to the test targets. For comparison, an automatic algorithm that estimated MSP based on symmetry was also implemented and tested. For fair assessment, the same initialization as discussed in Section 4.2 was performed before executing the symmetry-based algorithm.

To assess the accuracy of the algorithms, a human expert was asked to mark ground-truth landmark points on the skulls. Their mean distances to the detected planes were used to measure the identification error. This error measurement is consistent with the medical definition of the planes.
Table 1. Comparison of mean error. (full) Full test skulls. (partial) Partial skulls cut from full skulls. (patient) Patients' partial skull models. (ini) Initialization. (sym) The symmetry-based method. (prop) The proposed method.

\begin{tabular}{|c|c|c|c|c|c|}
\hline \multirow{2}{*}{$\begin{array}{c}\text { skull } \\
\text { type }\end{array}$} & \multicolumn{2}{|c|}{ FP (mm) } & \multicolumn{3}{c|}{ MSP (mm) } \\
\cline { 2 - 6 } & ini & prop & ini & sym & prop \\
\hline full & 1.17 & 0.64 & 1.47 & 0.86 & 0.48 \\
\hline partial & 1.26 & 0.60 & 0.97 & 0.79 & 0.50 \\
\hline patient & 1.72 & 0.58 & 1.17 & 1.01 & 0.61 \\
\hline
\end{tabular}

Fig. 3 and Table 1 show the results of the applied methods on target skulls. These results show that the proposed method can identify FP and MSP accurately and robustly. For FP identification, the proposed automatic method gives an error around $0.61 \mathrm{~mm}$ for all the three test cases, which is highly accurate compared to the CT resolution.

For MSP identification, the proposed method is also very accurate. It identified MSPs closer to the midline landmarks than did the symmetry-based method. The error of the proposed method is consistently lower than that of the symmetry-based method for all test cases.

In addition, the proposed method is robust to the asymmetry in skulls. Its accuracy on full and partial skulls are roughly the same $(0.48 \mathrm{~mm}$ and $0.50 \mathrm{~mm})$ because important landmark points can be located on them. The small increase in error for patient skulls is within an acceptable range because patient skulls have fractures.

In contrast, test results show that the symmetry-based method is less robust because it uses all the mesh vertices, most of which are outliers in defining MSP. Compared to partial skulls, full skulls have top and bottom parts, which tend to be outliers. Therefore, the symmetry-based method, using all the mesh vertices, has a larger error on full skulls. Patient skulls have more outliers than partial skulls due to fractures. Therefore, the error for patient skulls is the largest.

Overall, the proposed method is consistently accurate in identifying both FP and MSP on all kinds of skulls. Moreover, in identifying MSP, the proposed method, using only the midline features, is more accurate and robust than the symmetry-based method.

\section{Conclusion and Future Work}

This paper presented an automatic, robust, and accurate method for identifying FP and MSP of human skulls. The method registers a template skull model with known landmarks to the target skull to obtain good initialization of the landmarks, FP, and MSP on the target. Next, it iteratively refines the landmarks, FP, and MSP according to anatomical definitions. Test results show that the algorithm is more robust and accurate than a symmetry-based algorithm in identifying MSP. Moreover, it can be applied to partial skulls. 
(1)

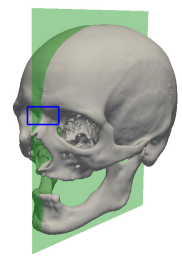

(2)

(3)

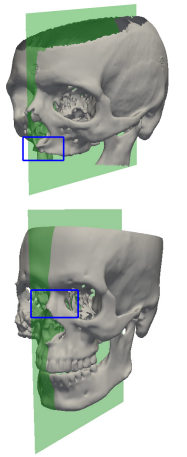

(a)

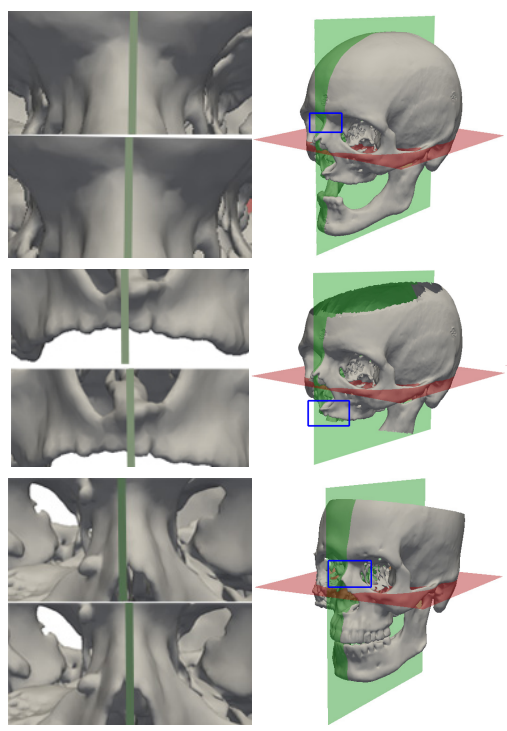

(b) (c)

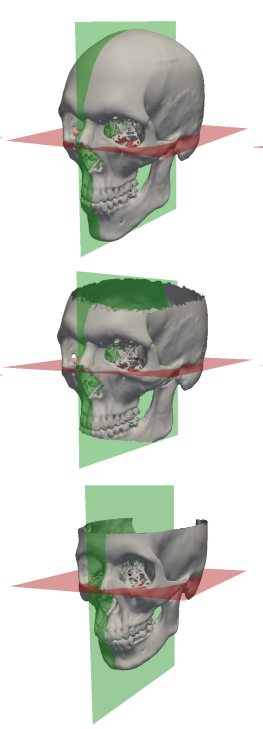

(d)

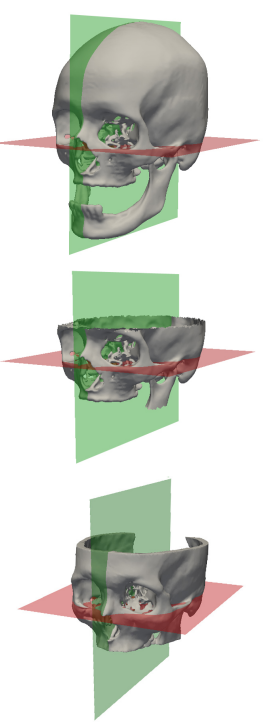

(e)

Figure 3. Identified MSP and FP. (1) Full skulls. (2) Partial skulls of (1). (3) Patient Skulls. (a) MSP of the models in (c) detected by symmetry-based method. (b) Zoom-in comparison of (a) on top and (c) at bottom. (c-e) Planes detected by proposed method.

At present, the method detects only FP and MSP landmarks. Our continuing research is to extend it to detect other important craniometric landmarks.

The proposed method works well for normal skulls and skulls with minor fractures. For certain plagiocephalic children and patients with serious head injuries, their skulls may be grossly distorted and the landmarks may not be located at their normal positions. In these cases, the proposed method is not expected to locate the landmarks accurately. As the skulls are grossly distorted, the concept of FP and MSP may not have much relevance in these cases, unless the surgeon wishes to perform surgical procedures to restore the patients' skulls to normal condition. Then, the FP and MSP of the restored skull may be compared to those of the template skull to assess the difference in alignment.

\section{References}

[1] D. Anderson, L. Anderson, and W. Glanze. Mosby's Medical Dictionary. Mosby St. Louis, 2009. 233, 234

[2] B. Avants and J. Gee. The Shape Operator for Differential Analysis of Images. In Proc. of Int. Conf. on Information Processing in Medical Imaging, pages 101-113, 2003. 236

[3] P. Besl and N. McKay. A Method for Registration of 3D Shapes. IEEE Trans. on Pattern Analysis and Machine Intelligence, 14(2):239-256, 1992. 235

[4] M. Cavalcanti, J. Haller, and M. Vannier. Three-Dimensional Computed Tomography Landmark Measurement in Craniofacial Surgical Planning: Experimental Validation in Vitro. $J$. Oral and Maxillofacial Surgery, 57(6):690-694, 1999. 233

[5] U. Clarenz, M. Rumpf, and A. Telea. Robust Feature Detection and Local Classification for Surfaces based on Mo- ment Analysis. IEEE Trans. on Visualization and Computer Graphics, 10(5):516-524, 2004. 236

[6] E. De Momi, J. Chapuis, I. Pappas, G. Ferrigno, W. Hallermann, A. Schramm, and M. Caversaccio. Automatic Extraction of the Mid-Facial Plane for Cranio-Maxillofacial Surgery Planning. Int. Journal of Oral and Maxillofacial Surgery, 35(7):636-642, 2006. 233, 234

[7] M. DoCarmo. Differential Geometry of Curves and Surfaces. Prentice-Hall, 1976. 236

[8] H. Gumprecht, D. Widenka, and C. Lumenta. BrainLab VectorVision Neuronavigation System: Technology and Clinical Experiences in 131 Cases. Neurosurgery, 44(1):97-104, 1999. 233

[9] H. Pancherz and K. Gokbuget. The Reliability of the Frankfort Horizontal in Roentgenographic Cephalometry. The European Journal of Orthodontics, 18(4):367-372, 1996. 233

[10] J. M. Phillips, R. Liu, and C. Tomasi. Outlier Robust ICP for Minimizing Fractional RMSD. In Proc. of Int. Conf. on 3D Digital Imaging and Modeling, pages 427-434, 2007. 235

[11] M. Rossi, E. Ribeiro, and R. Smith. Craniofacial Asymmetry In Development: An Anatomical Study. The Angle Orthodontist, 73(4):381-385, 2003. 233, 234

[12] D. Siwek and R. Hoyt. Anatomy of the Human Skull. http: / / skullanatomy.info. 233, 234

[13] K. Taylor. Forensic Art and Illustration. CRC, 2001. 233, 234

[14] S. Van Cauter, W. Okkerse, G. Brijs, M. D. Beule, M. Braem, and B. Verhegghe. 3D Cephalometry: A New Approach for Landmark Identification and Image Orientation. In Proc. of European Conf. of Int. Federation for Medical and Biological Engineering, pages 1442-1445, 2009. 233, 234

[15] T. Woo. On the Asymmetry of the Human Skull. Biometrika, 22(3/4):324-352, 1931. 233, 234 